\title{
EL AZAR EN LA MECÁNICA CUÁNTICA: DE BOHR A
} BELL

SERGio MarTinez MuNOZ

Instituto de Investigaciones Filos6ficas

UNAM

\section{Introducción}

Explicar el sentido en que el azar es objetivo, y no un mero reflejo de nuestra ignorancia, requiere la elaboración de una explicación del azar en términos de la estructura física del mundo, tal y como ésta se describe en nuestras teorías más exitosas. Aquí examino los fundamentos conceptuales de la mecánica cuántica con este proyecto en mente. Parto de un examen de la manera en la que Bohr entendía el origen físico del azar cuántico. Esta manera de entender el azar cuántico, que en lo esencial se encuentra implícita en exposiciones usuales de la teoría hasta el presente, presupone que el azar cuántico surge, como en el caso del lanzamiento de una moneda, de restricciones a nuestra capacidad de hacer mediciones de cierto tipo. Hago ver que esta presuposición es ilegítima. Posteriormente muestro que en el marco de una formulación lógico-algebraica de la teoría cuántica y de un concepto de estado de sistema individual desarrollado en detalle en otros trabajos, la distinción entre los principios de "separabilidad" y "localidad" nos lleva a la elaboración de un tipo diferente de respuesta.

No pretendo con este análisis agotar el tema de la objetividad del azar en la mecánica cuántica. Mi interés radica más bien en sugerir una manera de entender la objetividad o la irreduc- 
tibilidad del azar, como diremos indistintamente, en términos de la no-separabilidad de los estados cuánticos.

Es común pensar que la tesis del determinismo es equivalente a la tesis de que no hay azar físico irreductible. Sin embargo, la relación entre la tesis del determinismo y la tesis de la existencia de un azar físico irreductible es sutil. Por una parte, como John Earman hace ver en 1986, es posible encontrar modelos no deterministas de teorías de la mecánica clásica en los cuales el indeterminismo no proviene de la presencia de probabilidades. Por otra parte una serie de trabajos recientes de mecánica estadística y de teoría ergódica, entre los cuales los trabajos de Prigogine y sus colaboradores son quizás los más conocidos (por ejemplo, Misra et al., 1979, 1980), pretenden mostrar cómo probabilidades objetivas (irreductibles) y comportamiento azaroso de sistemas pueden surgir en el marco de una dinámica determinista. Por ello, es crucial para la tarea que me he propuesto en este trabajo que distingamos los diferentes tipos de azar independientemente, en lo posible, de la tarea de aclarar la tesis determinista. Empiezo haciendo algunas distinciones preliminares.

Me apartaré de la distinción tradicional entre azar epistémico y azar ontologico u objetivo. La idea de esta distincion es que el azar epistémico es el resultado de nuestra ignorancia de información o de condiciones objetivamente dadas, mientras que el azar ontológico u objetivo es parte de la realidad física. Esta distinción, que proviene de la interpretación clásica de las probabilidades formulada canónicamente por Laplace, la considero inapropiada para explicar las sutilezas de la noción de azar implícita en las teorías científicas de las que disponemos a finales del siglo XX.

Si bien considero que sería una tarea interesante y valiosa la presentación de una crítica detallada de la distinción tradicional entre azar epistémico y azar ontológico (objetivo), aquí simplemente parto de una manera diferente deconcebir la obje- 
tividad del azar. La justificación para establecer tal distinción está implícita en la discusión que sigue (véase además Martínez, 1990a). En primer lugar distingo entre azar puramente epistémico (no objetivo) y azar objetivo. Un azar puramente epistémico es el azar que se considera un producto de nuestra ignorancia de condiciones objetivas no azarosas "en principio". La interpretación clásica de las probabilidades consideraba que todo azar era de este tipo. A su vez, distingo dos tipos de azar objetivo: azar epistémico objetivo y azar sistémico objetivo. Un azar epistémico objetivo surge de las limitaciones que la estructura física del mundo impone en nuestras posibilidades de conocer esa estructura. Así, por ejemplo, si pensamos que las descripciones probabilistas de la mecánica clásica estadística son irreductibles porque (por lo menos en algunos casos importantes) la determinación de una trayectoria única requeriría una cantidad infinita de energía, entonces le estamos atribuyendo a estas descripciones probabilistas una objetividad epistémica. Este tipo de azar es el tipo de azar objetivo que Von Plato examina (1982). El azar es objetivo en tanto se asume que la existencia de una cantidad finita de energía es parte de la estructura física del mundo.

Una teoría fundamental de la física es una teoría que por medio de su descripción de estado describe completamente el tipo de sistemas a los que se refiere. Esto es, una teoría fundamental nu admite variables ocultas que permitan explicar un aspecto de la descripción de estado (y en particular una descripción probabilista) en términos de una descripción más detallada de la realidad física pertinente. Así, la mecánica clásica de partículas sería una teoría fundamental de las partículas clásicas (pero no de las partículas con comportamiento cuántico que ahora creemos que constituyen la materia), mientras que la mecánica estadística clásica, tal y como se interpreta usualmente no sería una teoría fundamental ya que la descripción 
de los sistemas estadísticos se basa en la ignorancia del estado preciso de los sistemas componentes. ${ }^{1}$

Un azar objetivo no epistémico tiene que expresarse como la descripción de un aspecto de la estructura física del mundo, incorporado en la descripción de estado, según una teoría fundamental de la física (independientemente de las limitaciones de nuestra manera de observarlo o conocerlo que puedan existir). A este tipo de azar objetivo le llamaremos azar sistémico. Es un tipo de azar objetivo que es intrínseco a la descripción de estado que la teoría utiliza. El tipo de azar objetivo que Prigogine atribuye a las descripciones de procesos estadísticos clásicos, en tanto que surgen supuestamente en el marco de una teoría fundamental, debería considerarse como un azar sistémico. Sin embargo, la aclaración del tipo de objetividad del azar en la teoría de Prigogine es un tema complicado que no puedo tratar aquí (refiero el lector interesado a Batterman, 1991). He sugerido (Martínez, 1990a, retomando una idea de Blatt, 1959) otra manera en que la mecánica estadística clásica puede interpretarse como describiendo un azar sistémico. Según esta interpretación, la mecánica estadística clásica describiría sistemas individuales que no son totalmente aislables del medio ambiente y de otros sistemas. Las probabilidades expresarían

1 Precisar la nocion de teoría fundamental requeriría adentrarnos en las sutilezas y dificultades del concepto de "teoría de variables ocultas". Para nuestro propósito presente es suficiente caracterizar a una teoría fundamental como aquella teoría $T$ que describe procesos azarosos, tal que no existe otra teoría $T^{*}$ que satisface ciertos requisitos físicos mínimas de adecuación en la cual los procesos en cuestión sean descritos deterministamente. Por supuesto, las dificultades surgen cuando se tratan de caracterizar tales requisitos mínimos.

En el caso de la mecánica cuántica, por ejemplo, estos requisitos mínimos incluirían alguna versión del principio de localidad. Precisamente qué versión del principio debe incluirse es tema de disputas. Un aspecto importante del problema de fondo en estas disputas tiene que ver precisamente con el tema de este trabajo, con la manera en la que la selección de los requisitos mínimos de adecuación nos permite explicar la naturaleza del azar cuántico. 
esta falta de distinción tajante entre un sistema y su "medio ambiente".

\section{La naturaleza del azar cuántico según Bohr}

Un problema que toda interpretación de la mecánica cuántica tiene que afrontar es el problema siguiente. Asumiendo que la mecánica cuántica es una teoría fundamental, icuál es el origen fisico de las descripciones azarosas de la teoría? Examinaremos en esta seccion, muy brevemente, cómo trat 6 Bohr de responderse a esta pregunta con base en un análisis del proceso de observación. Este tipo de respuesta del origen del azar cuántico es la respuesta asociada con la así llamada interpretación de Copenhague.

La interpretación de la mecánica cuántica de Bohr es un tema bastante complejo. Aquí no nos interesa elaborar las sutilezas y cambios de perspectiva que son evidentes cuando se estudian los escritos de Bohr a lo largo de los más de treinta años en los que se ocupó del problema. La interpretación de Bohr a la que aquí voy a referirme es sólo el núcleo de una interpretación que puede detectarse en sus escritos maduros y que ha sido muy influyente en el desarrollo de la interpretación ortodoxa. Hay una serie de interpretaciones que han surgido como variantes de la interpretación de Bohr; todas ellas sin embargo se caracterizan por dar esencialmente el mismo tipo de análisis del origen del azar cuántico. Es por ello que me concentro en un examen de la interpretación de Bohr y dejo de lado las diferentes variantes y complicaciones de las interpretaciones tradicionales como por ejemplo la interpretación de Heisenberg (que en otros aspectos es muy diferente de la de Bohr y es más cercana a las interpretaciones que aparecen en los libros de texto contemporáneos). ${ }^{2}$

2 En formulaciones posteriores de la interpretación de Copenhague, el llamado "principio de incertidumbre" substituye al postulado cuántico como expresión de las limitaciones objetivas en el proceso de medición 
Según Bohr, no es posible definir literalmente el estado clásico de un sistema físico por medio de observaciones, ya que la observación requiere una interacción con el sistema. Literalmente, en el momento de la observación el sistema no está aislado, una presuposición que la física clásica requiere implícitamente para definir el estado de un sistema. En el límite de mediciones que requieren poca energía con respecto a la cantidad medida, por ejemplo en el caso de la medición de la velocidad de un automóvil o de la masa de un planeta, podemos despreciar la perturbación causada por la medición del sistema. Esta imposibilidad literal de determinar simultáneamente los parámetros que caracterizan el estado (clásico) de un sistema, en conjunción con la hipótesis empírica del postulado cuántico, según el cual los estados de los sistemas cuánticos son discretos, llevan a interpretar las probabilidades generadas por el algoritmo cuántico como la expresión cuantitativa de la aplicación limitada del marco conceptual clásico al dominio microscópico. El núcleo de la interpretación de Bohr es este papel epistemológicamente privilegiado que le asigna a la física clásica con base en su análisis del proceso de la observación.

Hay dos maneras en las que podemos entender la naturaleza del azar cuántico en el marco de la interpretación de Bohr. Como veremos, es posible pensar que el tipo de azar cuántico implícito en las descripciones de estado que asumen el postulado cuántico es un azar epistémico objetivo. Éste es el tipo de azar que Bohr parece haberle atribuido a la mecánica cuántica. El postulado cuántico jugaría el papel de restricción objetiva al proceso de observación, a partir del cual se generan las descripciones azarosas características de la mecánica cuántica. Sin embargo, quiero sugerir que la hipótesis del postulado cuántico es compatible con una interpretación del azar cuántico como puramente epistémico. La primera manera de inter-

que generan las probabilidades cuánticas. Esta modificación no altera las conclusiones a las que llegamos más adelante. 
pretar la intuición básica de Bohr parece estar fundamentada en el análisis de casos típicos de procesos azarosos. Por ejemplo en el análisis del origen del azar en el caso del lanzamiento repetido de una moneda sobre una mesa. Si consideramos el azar en relación con nuestra capacidad de predecir el resultado del lanzamiento puede argüirse que el azar es epistémicamente irreductible en tanto que, por ejemplo, una predicción del resultado del lanzamiento requeriría una cantidad infinita de energía (véase por ejemplo Von Plato, 1982; Martínez, 1990a). ${ }^{3}$ El azar sería entonces epistémicamente irreductible, pero sistémicamente reductible. En el caso de la mecánica cuántica podríamos hacer un análisis similar. El postulado cuántico impondría un límite físico que no permitiría la predicción con certeza del resultado de una medición.

Esta interpretación de las probabilidades cuánticas presupone implícitamente la no existencia de una teoría de variables ocultas. No tendría mucho sentido interpretar las probabilidades cuánticas como irreductibles si no se excluyera la posibilidad de variables ocultas, pero Bohr no tiene un argumento separado en contra de la existencia de tales variables ocultas. Bohr parece creer que su análisis del proceso de observación apunta a las raíces físicas del azar cuántico i.e., al sentido en que las probabilidades cuánticas son irreductibles (véase Bohr, 1927, 1929). Esta interpretación, sin embargo, presupone que el azar implícitamente descrito por las probabilidades cuánticas surge, como en el caso del lanzamiento de una moneda, de la existencia de una diferencia cualitativa entre el nivel microscópico y el nivel macrosçópico, y además, que esta distinción cualitativa proporciona una guía epistemológica a la "localización" posible de las variables ocultas. Es sólo sobre la base de esta presuposición que Bohr puede entonces apelar al

3 Esta es una reconstrucción por medio de variables ocultas que s6lo recientemente ha sido posible elaborar con rigor. Véanse por ejemplo Keller, 1986, y Ford, 1983. 
"postulado cuántico" para excluir la posibilidad de variables ocultas por medio de un argumento cualitativo. El siguiente ejemplo hace ver que esta presuposición es insatisfactoria.

\section{Probabilidades clásicas no aproximables por medio de observaciones}

El tipo de irreductibilidad en teorías estadísticas clásicas ejemplificada en la irreductibilidad epistémica del azar que estaría detrás de la descripción probabilista del lanzamiento de una moneda, surge de la imposibilidad de observar con la precisión necesaria cierto tipo de sucesos. Se considera que esta irreductibilidad es objetiva en tanto que depende de una cierta hipótesis que impone limitaciones físicas a la precisión de cualquier proceso de medición. Existe sin embargo un tipo diferente de azar que no depende del posible grado de precisión de nuestras observaciones. Como lo muestra el siguiente ejemplo este tipo de azar puede surgir en un mundo determinista (i.e., en un modelo de una teoría determinista). Esto nos lleva a tener que considerar la posibilidad de que el azar cuántico tal y como Bohr lo concibe sea un azar puramente epistémico (producto de nuestra ignorancia).

Consideremos la siguiente situación que es compatible con la mecánica clásica celeste. Dos estrellas de igual masa giran alrededor de su centro de gravedad común, la ley de Newton de la gravitación implica que la órbita de las dos estrellas es un círculo y que las estrellas están siempre en los extremos de un radio. Sea $P$ el plano que contiene la órbita circular y $D$ el eje de simetría que es perpendicular a $P$. Un cometa con masa muy pequeña se mueve a lo largo de $D$. De las leyes de la mecánica celeste se sigue que esto es posible y que si el cometa empieza a moverse con una velocidad inicial a lo largo de $D$, entonces el cometa seguirá moviéndose de ida y vuelta a lo largo de $D$, cruzando el plano $P$ en ambas direcciones en su recorrido. 
Cerca de las dos estrellas hay un planeta habitado por astrónomos que han mantenido información cuidadosa de las apariciones del cometa cuando atraviesa el plano $P$. Las condiciones de observacion son tales que no es posible observar el cometa en otra posición. Podemos incluso asumir que los astrónomos tienen información de todas las veces que el cometa ha atravesado el plano $P$ y del tiempo exacto en el que esto ha sucedido. Esto es, podemos asumir que nuestras observaciones son todo lo precisas que querramos. Aun así, la información no tiene ningún orden en especial, es azarosa, y no puede utilizarse como base para predecir la próxima aparición del cometa. Ekeland desarrolla en 1984 este ejemplo para hacer ver que el azar en un mundo determinista no está restringido a microfen6́menos. Para nosotros este ejemplo es importante porque ejemplifica un tipo de azar objetivo y un tipo de probabilidades que son irreductibles epistémicamente, pero que son compatibles con la existencia de una teoría determinista de variables ocultas.

Supongamos que un día unos astrónomos se van en una nave espacial hasta una distancia considerable de su planeta y pueden observar la situación desde allí. En un acto genial uno de los astrónomos sospecha el origen del azar y desarrolla una teoría de la mecánica celeste esencialmente equivalente a la newtoniana. El astrónomo concluye que el origen del azar es la incompletitud del estado del cometa. Lo que es crucial, sin embargo, es que esta incompletitud de nuestro concepto de estado no puede inferirse de la situación epistémica. La diferencia importante en los dos conceptos de azar que hemos presentado (en el caso de la moneda y en el caso de las dos estrellas), es que, en el primer caso no podemos reducir el azar a una descripción determinista debido a la imposibilidad de preparar ciertos estados. En este sentido tenemos acceso epistémico a lo que consideramos que es el origen del azar. Sabemos que la dificultad consiste en la imposibilidad de hacer observaciones suficientemente precisas que nos permitirían preparar un estado con la 
precisión necesaria. En el segundo tipo de ejemplo, nuestra capacidad de observar y llegar a conocer las condiciones iniciales con cualquier grado de precisión no se restringe. En el caso del lanzamiento de una moneda las peculiaridades del proceso por medio del cual los resultados se determinan apunta al origen del azar. En el ejemplo de las dos estrellas el azar no se origina de esta manera. Por ello, es perfectamente posible que el azar en una teoría de la física, y en la mecánica cuántica en particular, no pueda explicarse a partir de hipótesis que, como el postulado cuántico (o el principio de incertidumbre de Heisenberg), imponen restricciones objetivas a las posibilidades de hacer mediciones de cierto tipo.

\section{La no existencia de variables ocultas según Bell}

Una nueva etapa en la aclaración de la tesis de la no existencia de las variables ocultas, y por lo tanto en la aclaración del concepto de azar objetivo en la mecánica cuántica, principia con los trabajos de Bell; éstos hacen énfasis en la relación entre la estructura probabilística y el tipo de predicciones de la teoría. Bell demuestra que las teorfas "locales" de variables ocultas son incompatibles con la estructura probabilísticapredictiva de la mecánica cuántica. Durante más de veinte años este resultado de Bell ha dado lugar a candentes debates, sobre todo en relación con el significado físico de la "localidad" en cuestión. En los últimos años, sin embargo, se ha logrado formular una distinción entre los diferentes principios que se ocultan detrás de la noción de localidad de Bell.

Antes de introducir esta distinción, es importante para mi proposito introducir una distinción previa. En la mecánica cuántica debemos hacer una distinción clara entre dos tipos de estado, por un lado tenemos los estados individuales, y por otro los estados estadísticos. ${ }^{4}$ Un estado individual es una des-

4 Es común confundir estos dos tipos de estado. Esta confusión parece tener su origen en la interpretación tradicional de las probabilidades 
cripción (un conjunto de enunciados) de las propiedades que son el caso para el sistema en una "situación" dada. El concepto de situación es un concepto semántico que cada teorfa debe especificar como parte de una interpretación deseada (intended interpretation). En la mecánica cuántica las situaciones están especificadas por los así llamados "procedimientos de preparación de estado". Como he hecho ver (Martínez, 1991a), estas situaciones pueden hacerse corresponder a retículos máximos booleanos en el retículo de las propiedades de sistemas mecánico-cuánticos. Esto contrasta con la idea usual que consiste en identificar el estado de una sistema cuántico con todos los valores propios de subespacios (correspondientes a propiedades del sistema). ${ }^{5}$ Estados estadísticos son los estados a los que las leyes dinámicas se aplican (deterministamente en el caso de la mecánica cuántica).

Una propiedad de un sistema es el valor de una magnitud que puede ser el caso en un momento dado. La velocidad, por ejemplo, es una magnitud que tiene diferentes valores posibles (números reales) en un sistema dado. Una propiedad es ocurrente si es parte de la descripción del estado en el que está el sistema (en un momento dado). El principio de la separabilidad dice que dos sistemas espacialmente separados son separables si los sistemas poseen estados (individuales) separados, esto es, si las propiedades ocurrentes en un sistema no dependen (para su ocurrencia) de las propiedades ocurrentes en el otro sistema. El principio de la localidad, por el contrario, se refiere a la naturaleza de los vínculos causales físicamente

generadas por el algoritmo cuántico como probabilidades de transición de superposiciones a vectores-estado. Esto oscurece la diferencia que según nosotros debe mantenerse entre vectores-estado y estados individuales.

5 En el lenguaje de la formulación lógico-algebraica de la teoría, la identificación usual es entre el estado individual de un sistema y el ultrafiltro (o filtro máximo) reticular generado por el subespacio unidimensional correspondiente al vector-estado en cuestion. En nuestro marco semántico los estados individuales se representan por ultrafiltros booleanos (i.e., por ultrafiltros en subretículos booleanos. 
posibles: el estado (individual) de un sistema puede ser cambiado únicamente por efectos locales (i.e., efectos propagados con velocidad finita). ${ }^{6}$

Una vez hechas estas distinciones podemos pasar al examen de un tipo de azar cuántico presentado por Bell en 1976, donde formula la condición que él llama de "localidad causal" como sigue:

$$
P(A(e)=j / \eta, \lambda)=P(A(e)=j / \lambda)
$$

en donde $A$ es una magnitud de un sistema físico en el suceso (punto espacio-temporal) $e, j$ es el valor de $A$ que es el caso en $e, \eta$ representa el valor de las variables ocultas en el cono de luz pasado de $e$ y $\lambda$ representa un conjunto arbitrario de fenómenos físicos fuera de ese cono de luz.

A continuación Bell muestra que la mecánica cuántica elemental (e incluso la teoría cuántica de campo relativista) no es local en el sentido de LC. El argumento es bastante simple. Parte de la observación de que de acuerdo con la mecánica cuántica la emisión de una partícula $\alpha$ por un núcleo particular es esencialmente impredecible. Supongamos que tenemos un aparato detector de partículas $\alpha$ que está fuera del cono de luz pasado (backward light cone). Puesto que la emision es esencialmente impredecible nunca podemos estar seguros de que detectaremo la emisión de una partícula dada. Todo lo que podemos hacer es asignar una probabilidad (diferente de 1) a la posibilidad de detección. Una vez establecido esto Bell considera la probabilidad condicional de la detección de la partícula en nuestro detector, dado que sabemos que la partícula fue detectada por otro detector en otro punto del espacio. La probabilidad de detección en nuestro detector original es ahora cero. Esto concluye el argumento que según Bell establece que la

6 Varios artículos relacionados con la formulación de esta distinción entre localidad y separabilidad, y de sus implicaciones para los problemas de interpretación de la teoría, pueden encontrarse en Cushing y Macmullin (comps.), 1989. 
mecánica cuántica es no local. La probabilidad pasa de ser un número diferente de cero a cero.

El argumento de Bell no puede estar del todo bien, porque si lo estuviera ninguna teorla que incluyera probabilidades irreductibles podria ser local, puesto que todo lo que presupone el argumento es la existencia de probabilidades irreductibles y la presencia de un principio de conservación que nos dice que una partícula no puede estar en dos puntos a la vez. Según Hellman (1982), el argumento de Bell confunde probabilidades físicas irreductibles con probabilidades epistémicas. Dada una nueva información de cierto tipo ajustamos nuestras probabilidades para reflejar el cambio de conocimiento, pero esto no tiene nada que ver con ningún tipo de acción a distancia. Hellman propone una serie de criterios para tratar de distinguir probabilidades epistémicas de probabilidades físicas que nos permitirfa delimitar el alcance del argumento de Bell y aclarar la relación entre el indeterminismo, producto de la existencia de probabilidades irreductibles, y la propiedad de la localidad causal definida por Bell. Aquí quiero proponer una manera diferente de formular la relación entre localidad y la presencia de probabilidades irreductibles que surge de una evaluación diferente del error en el argumento de Bell.

\section{No-separabilidad e irreductibilidad}

Muestro a continuación cómo puede formularse una relación clara entre la presencia de probabilidades irreductibles y la no-separabilidad en la mecánica cuántica, si tomamos como punto de partida no las probabilidades generadas por el algoritmo cuántico, sino las probabilidades de transición entre "estados relativos". Esto requiere un breve resumen de algo que he desarrollado en otra parte. En Martínez (1991a) he hecho ver cómo es posible recobrar la estructura de espacios de Hilbert, característica de la mecánica cuántica, a partir de la suposición de que los estados de sistemas individuales pueden represen- 
tarse por medio de los átomos de las álgebras máximas de Boole que representan las magnitudes del sistema físico en cuestión. Estos estados individuales están relacionados por medio de una función que describe la probabilidad de transición entre los diferentes estados en una situación física dada.

Si en la mecánica cuántica aceptamos la existencia de estados individuales relacionados por probabilidades de transición de la manera anteriormente descrita, entonces la no-separabilidad de la teoría puede expresarse en términos de cambios en las probabilidades de transición entre las propiedades descritas por los estados individuales (de subsistemas de un sistema compuesto) de manera totalmente análoga a como Bell arguye por la no localidad (en el sentido de LC). ${ }^{7}$ Aquí haremos esto claro con un ejemplo. Como hice ver (Martínez, 1990b), en el caso de un sistema compuesto $S=X+Y$, que podemos pensar concretamente como formado por dos partículas con una magnitud $M$ (spin) cuyos únicos valores son $+1,0$ y -1 en el que el spin total es 0 (en una situación del tipo EPR), hay propiedades del sistema $S$ que son descritas por proposiciones de la forma $A \vee B$, donde, por ejemplo, $A$ es una proposición que dice que la magnitud $M$ en el subsistema $X$ tiene el valor +1 y la magnitud $M$ en el subsistema $Y$ tiene el valor -1, y $B$ es una proposición que dice que la magnitud $M$ del subsistema $X$ tiene el valor -1 y la magnitud $M$ en el subsistema $Y$ es +1 . Antes de medir la magnitud $M$ en el subsistema $X$ hay una probabilidad diferente de uno de que la medición de $M$ en $X$ dé como resultado el valor +1 . Pero si la medición de la magnitud $M$ en el subsistema $Y$, que puede estar muy alejado en el espacio, nos da el valor -1 , entonces la probabilidad de que encontremos el valor +1 al medir $M$ en $X$ es 1 . Con anterioridad a la medición del subsistema $Y$ todo lo que podíamos atribuirle objetivamente a los subsiste-

7 Esta manera de expresar la no-separabilidad de los estados cuánticos la presento en Martínez, 1991 b. 
mas era una probabilidad diferente de uno de detectar el valor +1 (o-1).

De esto no se sigue que toda teoria con probabilidades irreductibles sea no-local, pero si se sigue que toda teorla con estructura de propiedades no booleana y con estados individuales relacionados por una función de probabilidad de transición es no separable. Notese que si partimos de la manera usual de interpretar las probabilidades generadas por el algoritmo cuántico, como probabilidades de transición de superposiciones a vectores estado, no podemos formular la conexión arriba expuesta entre la no-separabilidad y la presencia de una función que describe probabilidades (irreductibles) de transición entre estados individuales. En este caso, la presencia de aspectos epistémicos de la descripción estadística no permitirían la derivación de una conclusión acerca de la separabilidad de los estados individuales. La no-separabilidad de los estados estadísticos es no problemática en principio y puede analizarse fácilmente en términos de cambios en la información disponible a partir de la cual se calculan las probabilidades epistémicas (o parcialmente epistémicas).

\section{Conclusión}

Por lo general las discusiones usuales de la interpretación de probabilidades objetivas asumen una cierta concepción clásica de estado según la cual la situación física descrita por un estado coincide con el mundo físico total. Esto es, se asume que la estructura lógico-algebraica de las propiedades de sistemas físicos es booleana. En el caso de la mecánica cuántica, que tiene una estructura no booleana de propiedades, podemos entender la objetividad del azar como un resultado de la no-separabilidad de los estados cuánticos. 


\section{BIBLIOGRAFIA}

Batterman, R., 1991, “Randomness and Probability in Dynamical Theories: On the Proposals of the Prigogine School", Philosophy of Science, no. 58, pp. 241-263.

Bell, J.S., 1976, "The Theory of Local Beables", Epistemological Letters, no. 9, marzo, pp. 11-23. Reproducido en Bell, 1987.

-, 1987, Speakable and Unspeakable in Quantum Mechanics, Cambridge University Press, Cambridge England.

Bohr, N.,1927, “The Quantum Postulate and the Recent Development of Atomic Theory", en Bohr, 1934.

— 1929, "The Quantum of Action and the Description of Nature", en Bohr, 1934.

- 1934, Atomic Theory and the Description of Nature, Cambridge at the University Press.

Blatt, J.M., 1959, “An Alternative Approach to the Ergodic Problem”, Prog. Theor. Physics, vol. 22, no. 6, pp. 745-756.

Cushing, R., y E. McMullin (comps.), The Philosophy of Quantum Mechanics, Univ. of Notre Dame Press.

Earman, J., 1986, A Primer on Determinism, Reidel, Dordrecht.

Ford, J., 1983, "How Random Is a Coin Toss", Physics Today, abril, pp. $40-47$.

Hellman, 1982, "Stochastic Einstein-Locality and the Bell Theorems", Synthèse, no. 53, pp. 461-504.

Keller, J., 1986, “The Probability of Heads", Mathematical Monthly, no. 93, marzo, pp. 191-197.

Martínez, S., 1990a, "La objetividad del azar en un mundo determinista", Crítica, no. 65, agosto, pp. 3-21.

$\longrightarrow, 1990 b$, "Más allá de la presuposición newtoniana: propiedades genuinamente disposicionales en la mecánica cuántica", Crítica, no. 66, diciembre, pp. 25-37.

- 1991a, "Luders Rule as a description of Individual State Transformations", J. Phil. of Science, vol. 58, septiembre.

- 1991b, "From Mechanisms to non-Separability", Abstracts of the IX Congr. of Logic, Method. and Philosophy of Science, Uppsala, agosto.

Ekeland, I., 1984, Le Calcul, l'Imprevu: Les Figures du temps de Kepler d Thom., Editions du Seuil. 
Plato von, J., 1982, "Probability and Determinism", J. Philosophy of Science, vol. 49, pp. 51-66.

Misra, B., I. Prigogine y M. Courbage, 1979, "From Deterministic Dynamics to Probabilistic Descriptions", Physica, A 98A, pp. 126.

Misra, B., y I. Prigogine, 1980, “On the Foundations of Kinetic Theory", Supplement of the Progress of Theoretical Physics, no. 69, pp. 101-110.

Racibido: 6 de septiembre de 1991 . 
Usual interpretations of the quantum theory, beginning with Bohr's interpretation, consider that randomness in quantum mechanics arises from (objective) restrictions to our capacity of making precise measurements of the corresponding state variables. I show that this assumption is problematic and leaves open important questions concerning the role and nature of quantum randomness. On the basis of a concept of state introduced elsewhere and on the basis of a distinction between the principles of separability and locality in quantum mechanics, I suggest in this paper a different sense in which quantum randomness is objective. In order to carry out the proposed task a distinction is made between two different types of objective randomness. Objective epistemic randomness arises from the limitations that the physical structure of the world imposes upon our possibilities of coming to know (measure) this structure. Objective non-epistemic randomness or systemic randomness is to be expressed in terms of a state description in a fundamental theory of physics. It is shown that the non-Boolean structure of properties of quantum theory, as this structure has been constructed and interpreted in Martínez 1991a, can be interpreted as an expression of the non-separability of the theory. Thus, the irreducibility of the transition probabilities that generate the non-Boolean structure of properties can be interpreted in this way as systemic randomness. In the case of quantum mechanics systemic randomness and non-separability are two sides of the same coin. 